Communications in Physics, Vol. 24, No. 3 (2014), pp. 225-231

DOI:10.15625/0868-3166/24/3/3791

\title{
SENSITIVE ORGANOPHOSPHORUS AND CARBAMATE PESTICIDES BIOSENSOR BASED ON ACETYLCHOLINESTERASE AND CdZnSe/ZnS TERNARY ALLOY QUANTUM DOTS
}

\author{
NGUYEN NGOC HAI, NGUYEN HAI YEN, DUONG THI GIANG, NGUYEN DUC NHAT, \\ PHAM THU NGA, AND DAO TRAN CAO \\ Institute of Materials Science, Vietnamese Academy of Science and Technology
}

E-mail: haidhsphn@gmail.com

Received 07 April 2014

Accepted for publication 30 June 2014

\begin{abstract}
This report presents the results of ternary alloy quantum dots (TQDs) manufacturing and its superior optical properties to fabricate the biosensor for pesticide detection. The explanation for the changes in PL intensity based on the change of electronic charges that alters the $\mathrm{pH}$ value of the surrounding environment, and the types of interaction on the surface of $C d Z n S e / Z n S T Q D$ s were discussed. We show the decomposition process of the indicator (ATCh) with the catalysis from acetylcholinesterase enzyme (AChE) that inhibited by organophosphorus and carbamate pesticides including Trichlorfon (TF), Cypermethrin (CH), Abamectin (AT), and Carbosulfan (CS) with concentrations from 0.05 ppm to 10 ppm.
\end{abstract}

Keywords: pesticide, biosensors, ternary alloy quantum dots, photoluminescence.

\section{INTRODUCTION}

Organophosphorus (OP) compounds, carbamates, or neonicotinoids are widely used in plant protection because of the efficiency for insect killing, easy synthesis, and low cost. The above pesticide residues are harmful to the environment, human health, and animals through the nervous system poisoning by the way of inhibiting the activity of AChE, the most important enzyme involved in nerve transmission $[1,6]$. The principal role of AChE is the termination of nerve impulse transmission at cholinergic synapses by rapid hydrolysis of the neurotransmitter acetylcholine (ACh) - each molecule of AChE degrades about 25000 molecules of ACh per second, leading to in vivo accumulation of $\mathrm{ACh}$, and then resulting in serious impairment on nerve functions [2].

TQDs with core/shell structure $\mathrm{Zn}_{x} \mathrm{Cd}_{1-x} \mathrm{Se} / \mathrm{ZnS}$ was fabricated and study on the structural characterization using several methods such as composition analysis with energy dispersive $\mathrm{x}$-ray spectroscopy (EDS) and transmission electron microscopy (TEM). The absorption and PL spectra and exciton lifetimes were also investigated to confirm the superior of this $\mathrm{CdZnSe/ZnS} \mathrm{TQDs}$ to binary composition quantum dots such as CdSe or CdTe, with the emission depending only on the size. For emission in the green $(\sim 520 \mathrm{~nm})$ or blue light, the size is relatively small, so 
that quantum dots would not durable [7]. TQDs' emission in green or blue region is more stable, easier to manufacture with large size, and the essential advantage in using them as the emission substance to create biosensors [8].

\section{EXPERIMENTAL}

The TQDs cores were synthesized with a constant precursor ratio of $\mathrm{Cd}_{0.2} \mathrm{Zn}_{0.8} \mathrm{Se}$. This method is adapted from existing work for CdSe quantum dots [1]. We used ultra-pure nitrogen flow for all synthetic routes. The molar ratio of the precursors for a typical synthesis of $0.5 \mathrm{mM}$ $\mathrm{Cd}_{0.2} \mathrm{Zn}_{0.8}$ Se alloy nanocrystal QDs is $\mathrm{Cd} / \mathrm{Zn} / \mathrm{Se}=0.2 / 0.8 / 3.33 ; \mathrm{TOPO} / \mathrm{HDA}=55 / 45$. Firstly, injecting TOP-Se precursor into the flask under vigorous stirring at temperatures of $100^{\circ} \mathrm{C}$ and heating up to $190^{\circ} \mathrm{C}$. After injecting TOP-Zn, the temperature was raised to $270^{\circ} \mathrm{C}$ and TOP-Cd precursor was injected. The nucleation of CdZnSe TQDs cores starts shaping quickly at $260^{\circ} \mathrm{C}$. At $280-290^{\circ} \mathrm{C}$ for 20 minutes, we obtain $\mathrm{Cd}_{0.2} \mathrm{Zn}_{0.8}$ Se alloy QD core. Secondly, synthesis of a $\mathrm{ZnS}$ shell on the $\mathrm{Cd}_{0.2} \mathrm{Zn}_{0.8}$ Se core. We evacuated the mixture of $0.25 \mathrm{mM} \mathrm{CdZnSe}$ TQDs and TOPO and HDA for an hour at $50^{\circ} \mathrm{C}$. The shell growth was done at a temperature of $220^{\circ} \mathrm{C}$ and the amount of $\mathrm{S}$ is $0.115 \mathrm{ml}$. The shell thickness depends on the amount of $\mathrm{Zn}$ precursor and $\mathrm{S}$ solvent. In this experiment, it is approximately 2, 4, 6 monolayers (ML) [3,4]. AChE enzymes (EC 3.1.1.7, activity unit: $500 \mathrm{U} / 0.7 \mathrm{mg}$ ) and S-acetylthiocholine iodide (ATChI) is purchased from Sigma-Aldrich. The ATCh was prepared from ATChI [6]. The four types of pesticides: TF; CH; AT; CS were obtained from Vietnam Plant Protection Department (PPD). The TQDs were surface-modified by MPA in order to assure water solubility and streptavidin (SA) molecules were attached to the TQDs [3]. Based on coulomb interaction, the AChE was conjugated to form sensitive TQDs-SA/AChE biosensors. TQDs-SA and AChE was mixed and incubated at $37^{\circ} \mathrm{C}$ for 10 minutes. Different amounts of the pesticides were added to the same corresponding amount of ATCh $(2 \mu \mathrm{mol})$, and the mixture is added to the biosensor solution, incubated at $37^{\circ} \mathrm{C}$ for $20 \mathrm{~min}$. The $\mathrm{pH}$ values of the initial solutions are around 7.1-7.3.

The optical properties of the TQDs were determined by the transmission electron microscopy (TEM), a JEOL Jem 1010 microscope system. PL measurements by laser excitation at $325 \mathrm{~nm}$. The PL decays were analyzed with a PM Hamamatsu R5600U and a Tektronix TDS 784A scope with a time constant of the order of $1 \mathrm{~ns}$. The low-temperature experiments were carried out in a Janis Supertran-VP with a variable temperature controlled between $4 \mathrm{~K}$ and $300 \mathrm{~K}$. Energy dispersive X-ray spectroscopy (EDS) have been used to determine the alloy crystal phase and composition of the TQDs.

\section{RESULTS AND DISCUSSION}

Fig. 1 shows the normalized PL spectrum with the excitation wavelength at $405 \mathrm{~nm}$, the FWHM is about $46.5 \mathrm{~nm}$, emission peak at $579 \mathrm{~nm}$ in the visible region and the inset above is the TEM images of $\mathrm{Cd}_{0.2} \mathrm{Zn}_{0.8}$ Se TQDs showing the spherical shape and the average sizes of TQDs core grown for $20 \mathrm{~min}$ are measured to be about 6.88-8.67 $\mathrm{nm}$. The TQDs create the large surface area for conjugating of biology agents to the TQDs [4].

Moreover, one of the criteria for the biosensor fabrication based on quantum dots is minimizing blinking effect. This directly affects the "in" and "out" signals of the quantum dots act as the transducers [7]. For the CdZnSe/ZnS 4ML sample, the time traces and the blinking are quite 


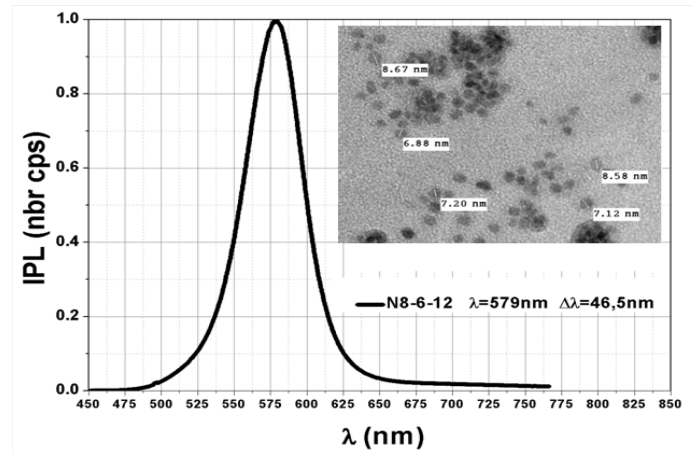

Fig. 1. Normalized PL spectrum $\left(\lambda_{e x c}=405 \mathrm{~nm}\right)$ and TEM images (inset above) of the $\mathrm{Cd}_{0.2} \mathrm{Zn}_{0.8} \mathrm{Se}$ cores

better to the $\mathrm{CdSe} / \mathrm{ZnS}$ nanocrystals. We do the same for 25 nanocrystals. For each nanocrystal, we define manually a threshold above which we consider that the nanocrystal is in the ON state, indicated by the curve in the Fig. 2. We selected four nanocrystals with these thresholds: 5600, $3000,1800,5300$, and then we calculated the portion of time spent in each nanocrystal in the ON state.

We calculated that, on average, the nanocrystals spend 35\% in the ON time. More specifically, about one third of them are in the ON state more than $50 \%$ of the time, and about one third of them are in the ON state less than $20 \%$ of the time $[6,7]$.

The composition of the TQDs samples is measured by energy dispersive X-ray (EDS) (Fig. 3). EDS analysis indicates that subsequent incorporation of zinc into the crystal lattice takes place after reaction times $(\geq 18 \mathrm{~min})$ and formation of $\mathrm{Cd}_{0.2} \mathrm{Zn}_{0.8}$ Se alloy nanocrystals is observed. Elemental analysis of ensembles of the TQDs reveals the presence of $\mathrm{Zn}, \mathrm{Cd}$, and Se for the TQDs core and the presence of the $\mathrm{Zn}$ and $\mathrm{S}$ elements in the shell. Based on the superior optical properties, alloy crystal structure, the $\mathrm{Cd}_{0.2} \mathrm{Zn}_{0.8} \mathrm{Se} / \mathrm{ZnS} 6 \mathrm{ML}$ TQDs were selected for the biosensor fabrication.

AChE enzyme catalyzes the hydrolysis of ATCh to produce TCh, which bears an additional thiol group (-SH) to possibly decrease the $\mathrm{pH}$ surrounding the TQDs. Fig. 4 shows the result of specific amount of $100 \mu 1 \mathrm{TQDs}, 122 \mu \mathrm{l} \mathrm{AChE}$ and $16.7 \mu \mathrm{l}$ ATCh, we observed the PL intensity of TQDs/AChE/ATCh solution decreased, and it takes $\sim 12$ min to reach the stable value.

In this biosensor structure, ATCh acts as an indicator for the presence of the AChE enzymatic activity, while the TQDs act as the luminescence indicators for the hydrolysis of ATCh. Eventually, the luminescence from TQDs can indicate the AChE enzymatic activity or correspondingly the pesticide content because of adding pesticide into the mixed solution. The pesticide would bind and inhibit the AChE enzymatic activity. Base on the mechanism, four types of organophosphorus (Trichlorfon-TF, Cypermethrin-CH); carbamate (Abamectin-AT, CarbosulfanCS) pesticides were detected. Fig. 5 presents the same trend of PL intensity increase with the concentration at $0.05 ; 2.0 ; 5.0 ; 8.0$ and $10.0 \mathrm{ppm}$. (The EU standard is $0.05 \mathrm{ppm}$ for the most of agricultural products) [5]. 

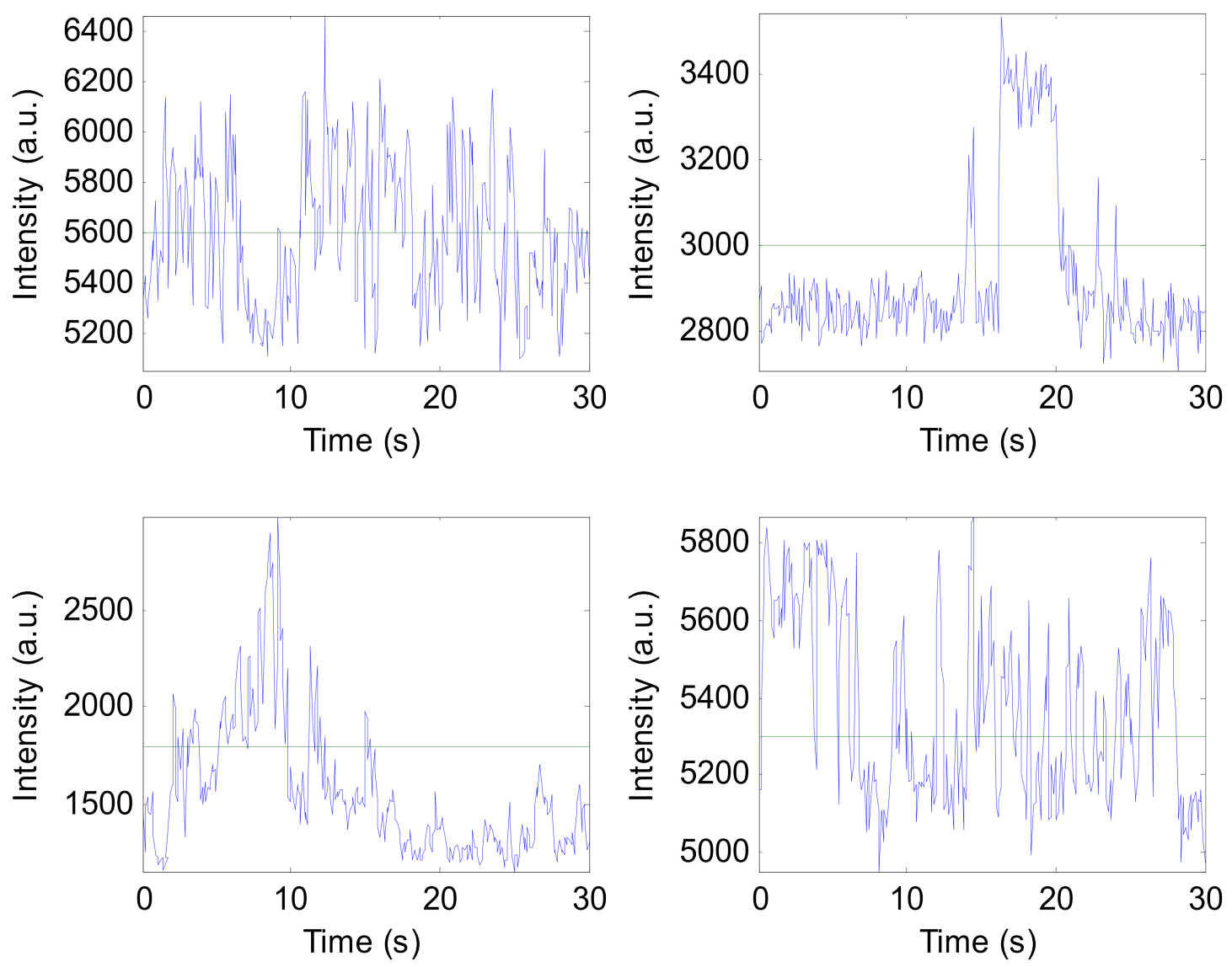

Fig. 2. Intensity time traces of four different nanocrystals, measured by CCD camera with a $100 \mathrm{~ms}$ resolution for sample CdZnSe/ZnS 4ML

The $\mathrm{pH}$ values were measured at the temperature of $31.5-32{ }^{\circ} \mathrm{C}$. The minimum $\mathrm{pH}$ value of TQDs/AChE/ATCh is about 5.5 and increased with increasing concentrations of pesticides until reaching the maximum $\mathrm{pH}$ value of the mixture TQDs/AChE initially about 7.1-7.2. The PL intensity (I) of the corresponding concentrations is compared to the one of TQDs/AChE/ATCh $\left(I_{o}\right)$; the maximum ratio is 1.52 for the case of TF at concentration of $10.0 \mathrm{ppm}$. (Table 1 ).

Table 1. The PL intensity ratio ( $\left.\mathrm{I} / \mathrm{I}_{o}\right)$ of corresponding pesticide concentrations (C).

\begin{tabular}{cccccccc}
\hline $\mathbf{C}(\mathrm{ppm})$ & 0.0 & 0.05 & 2.0 & 5.0 & 8.0 & 10.0 & Pesticides \\
\hline \multirow{3}{*}{$\mathbf{I} / \mathbf{I}_{o}$} & 1.0 & 1.22 & 1.38 & 1.48 & 1.50 & 1.52 & $\mathrm{TF}$ \\
\cline { 2 - 8 } & 1.0 & 1.30 & 1.31 & 1.35 & 1.47 & 1.56 & $\mathrm{CH}$ \\
\cline { 2 - 8 } & 1.0 & 1.12 & 1.23 & 1.35 & 1.46 & 1.58 & AT \\
\cline { 2 - 8 } & 1.0 & 1.02 & 1.04 & 1.07 & 1.24 & 1.30 & $\mathrm{CS}$ \\
\hline
\end{tabular}




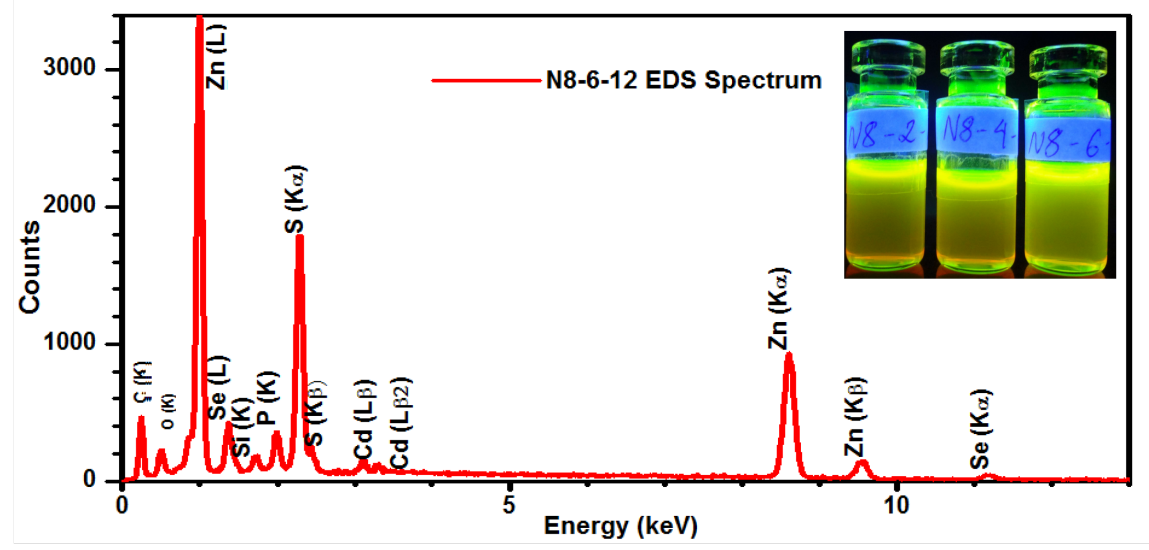

Fig. 3. EDS spectrum $\mathrm{Cd}_{0,2} \mathrm{Zn}_{0,8} \mathrm{Se} / \mathrm{ZnS} 6 \mathrm{ML}$

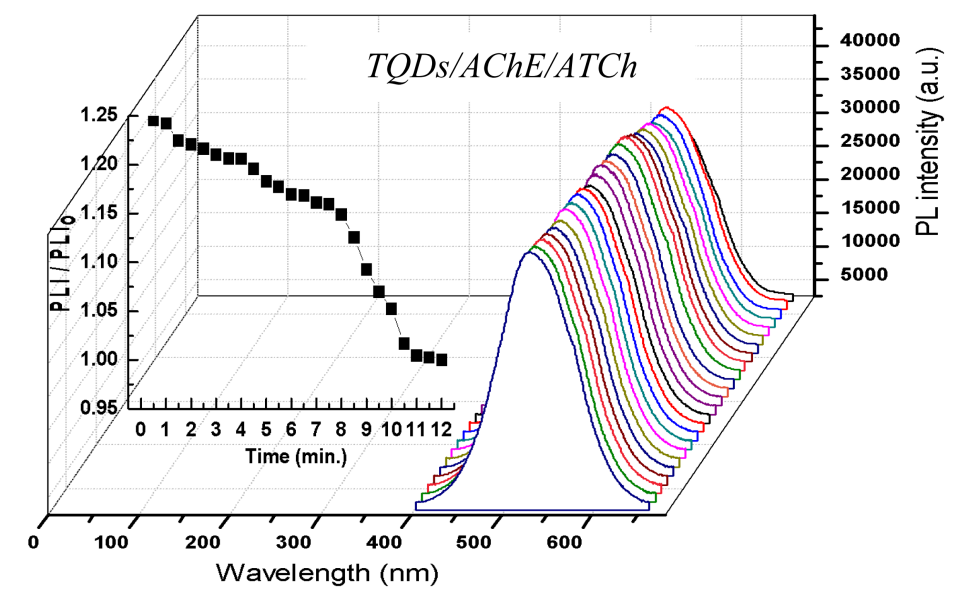

Fig. 4. The changes in fluorescence intensity over time of TQDs /AChE/ATCh

Fig. 6 shows the integrated PL intensity that increasing with the accreting of pesticides content. The detection range can be expandable by increasing the AChE content (also meaning the increase of the AChE: TQDs ratio) [3].

The pesticides might interact with the activity center of AChE preventing from acetic acid production. In contrast, if the number of protons $(\mathrm{H}+)$ generated more by acetic acid $(\mathrm{pH}$ decreased), the $\mathrm{H}+$ can capture the electron $\left(\mathrm{e}^{-}\right)$on the surface of the TQDs so that decreasing the e-h recombination in TQDs and causes PL quenching of the biosensor [4,7]. For quantum dots passivity with an inorganic capping layer, the TQDs core is often assumed isolated from the outside environment, leading to the expectation that the TQDs photoluminescence should not be affected by external factors. However, MPA-capped core/shell TQDs is highly sensitive to $\mathrm{pH}$, with greater 

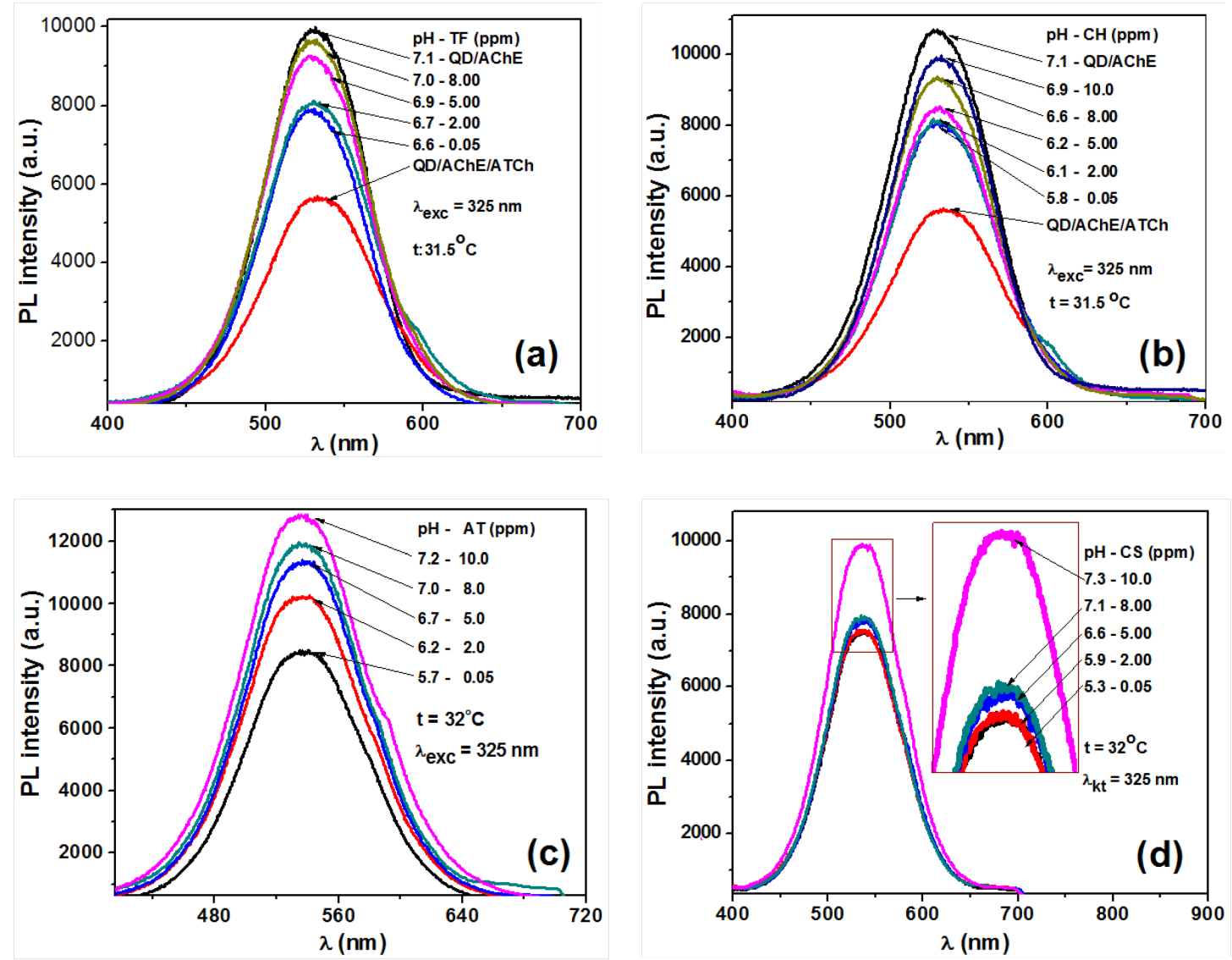

Fig. 5. Fluorescence spectra of the biosensor (TQDs / $\mathrm{AChE}$ ) with $\mathrm{TF}(\mathrm{a}), \mathrm{CH}(\mathrm{b}), \mathrm{AT}(\mathrm{c})$, CS(d) concentrations: $0.05 ; 2 ; 5 ; 8 ; 10 \mathrm{ppm}$

fluorescence at higher $\mathrm{pH}$, and a small red shift in the peak emission with increasing $\mathrm{pH}$. This increase of fluorescence intensity with increasing $\mathrm{pH}$ has also been reported for $\mathrm{CdSe} / \mathrm{ZnS}$ binary quantum dots [8].

\section{CONCLUSION}

TQDs of CdZnSe/ZnS have been successfully fabricated with less blinking effect, large surface, and emitting the light in the visible region but has a surface area large enough to conjugate with biological agents to form the biosensor. It was confirmed that the TQDs emission depends not only on the particle size but also the components percentage. In fact, the experiments were carried out to detect four pesticides (OP and carbamate). The results showed that the PL intensity increased with increasing pesticide concentrations in the range from 0.05 to $10 \mathrm{ppm}$. 


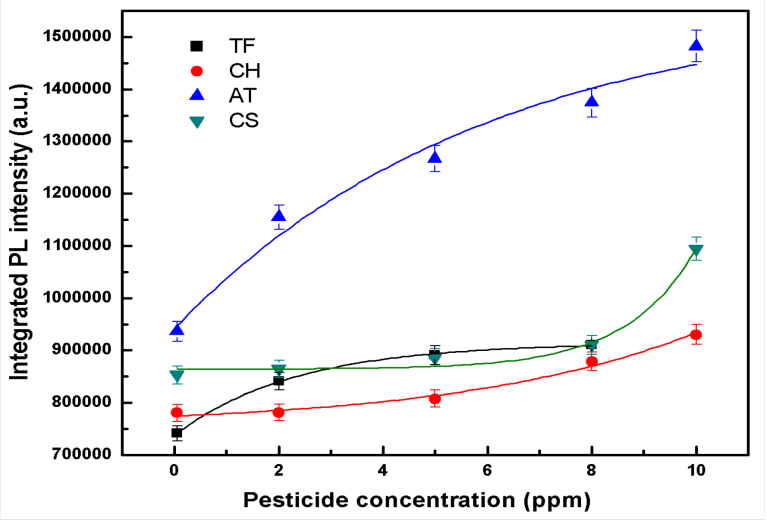

Fig. 6. The dependence of the fluorescence intensity on the concentration of 4 types of pesticides: TF, CH, AT, CS

\section{ACKNOWLEDGMENT}

This research is funded by NAFOSTED under grant number 103.06-2011.03, the PICS$\mathrm{n}^{\circ} 5724$ and the support for young researcher from IMS-VAST. The authors thank the National Key Laboratory for Electronic Materials and Devices-IMS for the supplying the facilities. We sincerely thank Prof. Nguyen Quang Liem for his judicious discussions.

\section{REFERENCES}

[1] Z. Deng, H.Yan, and Y. Liu, J. Am. Chem. Soc. 131 (2009) 17744-17745.

[2] Chen Y., Vela J et al, Journal of the American Chemical Society. 130 (2008) 5026-5027.

[3] Q. Chen, Y. Fung, Electrophoresis. 31 (2010) 3107-3114.

[4] Z. Zheng, Zhou Y., Li X., Liu S., and Tang Z, Biosensors and Bioelectronics. 26 (2011) 3081-3085.

[5] T.T.K.Chi et al, Adv. Nat. Sci.: Nanosci.Nanotechnol. 3 (2012) 035008.

[6] P.T. Nga, V.D. Chinh, V.T.H. Hanh, N.X. Nghia and P.T. Dzung, Int. J. Nanotechnol. 8 (2011) 347-359.

[7] Mahler B., Spinicelli P., Buil S., Quelin X., Hermier J. P., Dubertret B, Nat Mater 7 (2008) 659-664.

[8] N.N. Hai, V.D. Chinh , T.K. Chi , U.T.D. Thuy , N.X. Nghia, D.T. Cao, P.T. Nga, Key Engineering Materials. 495 (2012) 314-318.

tEST 